\title{
The Active Frequency Control Strategy of the Wind Power Based on Model Predictive Control
}

\author{
Ya-ling Chen, ${ }^{1}$ Yin-peng Liu $\mathbb{D}^{2},{ }^{2}$ and Xiao-fei Sun $^{3}$ \\ ${ }^{1}$ Business School of Hunan University, Changsha, Hunan, China \\ ${ }^{2}$ Institutes of Science and Development, Chinese Academy of Sciences, Beijing, China \\ ${ }^{3}$ School of Electrical Engineering, Southwest Jiaotong University, Chengdu, Sichuan, China \\ Correspondence should be addressed to Yin-peng Liu; liuyinpeng@casisd.cn
}

Received 11 August 2020; Revised 17 September 2020; Accepted 11 May 2021; Published 27 May 2021

Academic Editor: Qiang Chen

Copyright ( $\odot 2021$ Ya-ling Chen et al. This is an open access article distributed under the Creative Commons Attribution License, which permits unrestricted use, distribution, and reproduction in any medium, provided the original work is properly cited.

In this paper, an active frequency control strategy of wind turbines based on model predictive control is proposed by using the power margin of wind turbines operating in load shedding mode. The frequency response model of the microgrid system with the load shedding of the wind turbines is used to predict the output power and system frequency deviation of the wind turbine. According to the prediction information, the output power control signal of the model predictive controller in the wind turbine can be optimized. On this basis, a wind turbine active participation frequency control strategy based on model predictive control is designed by rolling prediction and optimization. The wind turbine power control signal after the strategy is used to adjust the output power of the wind turbine and balance the change of the active power of the system to reduce the frequency deviation.

\section{Introduction}

With the large-scale development and access of domestic wind turbines, some wind turbines already have frequency modulation (FM) capacity, so that they can actively participate in the FM control [1-3]. Therefore, further exploration and research of wind turbines piconets frequency controller design scheme, on the one hand, make full use of the frequency modulation capability of synchronous generators in the system to maintain the frequency stability of microgrid after wind turbines are connected; on the other hand, by introducing the active frequency control, the wind turbines effectively participate in the system dynamic frequency regulation, so as to further improve the frequency response of microgrid containing wind turbines, which is of great significance to ensure the safe and economic operation of microgrid $[4,5]$.

As wind power retains a significant proportion of generation mix in the electric system, it may impact the system's frequency security due to the lack of frequency support from units. To make up for such a system change, wind turbines should actively provide frequency response upon request. Hence, the variable speed wind turbine generators, such as doubly-fed induction generator (DFIG) [6] and direct-drive permanent magnetic synchronous generator (PMSG) [7], are widely studied for their great control characteristics in recent works of literature. The results of a study on the frequency deviation of the utility grid due to wind power fluctuations are presented in [8], in which the deviation is estimated by a deterministic method based on the transfer functions of system components. In [9], a cooperative control scheme for wind generators and plug-in hybrid electric vehicles (PHEVs) is proposed, in which the centralized and distributed control structures are considered and examined as two possible coordination methods. Ref. [10] proposes a primary frequency response strategy for the DFIG to regulate the remote area power supply (RAPS) system frequency and further proposes a supplementary control loop to enhance the primary frequency response with torque feedback control. The authors in [11] discuss the possibility of deloading wind turbines for frequency response using blade pitch angle control, in which a pitch control scheme is proposed to provide frequency response utilizing fixed-speed induction generator- (FSIG-) 
and DFIG-based wind turbines. In [12], authors explore several operating strategies for DFIGs to support system frequency with less sacrifice in the wind energy production and validate the effectiveness of the methodology in regulating wind power upon the operator's request, while stability is assured under variant wind speeds. In [13], a switching angle controller (SAC) and an automatic generation controller (AGC) for the DFIG are proposed to control the frequency of DFIG-based wind power penetrated power systems, in which the concept of virtual rotor angle of the DFIG is defined, and the virtual rotor angle is controlled by the SAC in a bang-bang manner. Besides, some other related works have been reported recently, such as learning control methods [14], USDE-based sliding mode control, and output feedback control $[15,16]$. However, in these above works, the load shedding for wind turbines is not considered, and the prediction of wind power is not discussed.

Therefore, considering both the load shedding and the prediction of wind turbines, this paper proposes a novel active frequency control strategy of the wind power based on model predictive control (MPC). The contributions of this paper can be summarized as follows:

(1) The load shedding operation model of the wind turbine is established in this paper, in which a certain amount of mechanical power reserve for frequency modulation is obtained by reducing load and maintaining wind turbines worked at a suboptimal operating point.

(2) An MPC-based active frequency control strategy is proposed for wind turbines in the paper by utilizing the power margin of wind turbines operating in load shedding mode, which is able to regulate the frequency deviations to be within the safe operation range and has the excellent dynamic performance of the system frequency.

(3) The proposed control strategy in the paper can make full use of the advantages of model predictive control. Specifically, the proposed strategy is feasible without wind speed prediction due to the power output delay caused by the inertia link of the wind turbine.

The remainder of this paper is organized as follows. Section 2 introduces the load shedding operation model of the wind turbine. In Section 3, the wind turbine active participation in a microgrid frequency control strategy based on model predictive control is introduced. Then, simulation results carried on a single-area and a four-zone power system are shown in Section 4. Finally, Section 5 concludes this paper.

\section{Load Shedding Operation Model of Wind Turbine}

Traditional wind turbines operate in the maximum power tracking mode, in which there is no power reserve for continuous frequency adjustment. In order to obtain a certain amount of mechanical power reserve for frequency modulation, wind turbines need to reduce load and maintain it at a suboptimal operating point, i.e., the load shedding operation model of the wind turbine. Specifically, this section describes the load reduction operation of the wind turbine to obtain the power reserve for frequency modulation.

The mechanical characteristic $P_{\mathrm{m}}\left(\omega_{r}\right)$ of the wind turbine is shown in Figure 1, wherein the solid gray curve represents the typical maximum power tracking curve of the wind turbine. In order to realize the wind turbine participating in the frequency regulation of microgrid, the sufficient mechanical power load reduction capacity of the wind turbine is required. Therefore, for the wind turbine in a maximum power tracking mode, there is no available power reserve for frequency control. However, adopting blade variable torque Angle control requires external mechanical functions, which is inflexible and may lead to mechanical wear problems. Therefore, in the following context, this section discusses the use of rotor speed control to obtain the power reserve.

As shown in Figure 1, for the rotor speed control mode, the operating point of the wind turbine can be moved from the maximum power tracking curve to its left or right side. This section uses the right shift to maintain a higher rotor speed and store additional kinetic energy to achieve shortterm frequency regulation. Therefore, the aerodynamic coefficient $C_{p}\left(\lambda_{\text {opt }}\right)$ is multiplied by a load reduction coefficient $k_{\text {del }}$ :

$$
C_{p}\left(\lambda_{\text {del }}\right)=k_{\text {del }} C_{p}\left(\lambda_{\text {opt }}\right)
$$

The active power of the wind turbine under load shedding mode is as follows:

$$
P_{\text {mdel }}\left(w_{\text {rdel }}\right)=k_{\text {del }} P_{\max }=0.5 \pi \rho R^{2} v_{w}^{3} C_{p}\left(\lambda_{d l}\right) .
$$

Under the load-shedding operation mode, the tip speed ratio can be deduced as follows:

$$
\lambda_{d e l}=-k_{1} \frac{1+\sqrt{\left(1-k_{d e l}\right)\left(1-4 k_{2} k_{0} / k_{1}^{2}\right)}}{2 k_{2}}=\lambda_{\mathrm{opt}} C_{d e l} \text {, }
$$

where the suboptimal coefficient $C_{d e l}$ is expressed as

$$
C_{d e l}=1+\sqrt{\left(1-k_{d e l}\right)\left(1-\frac{4 k_{2} k_{0}}{k_{1}^{2}}\right)}
$$

The load shedding suboptimal power tracking curve shown in the red line in Figure 1 and the load shedding rotor speed are shown as follows:

$$
\begin{aligned}
P_{\text {del }}\left(w_{\text {rdel }}\right) & =\frac{k_{\text {del }} k_{\mathrm{opt}}}{C_{\text {del }}^{3}} \omega_{\text {rdel }}^{3}, \\
\omega_{\text {rdel }} & =\frac{p k_{g} \lambda_{d e l}}{2 R} v_{w} .
\end{aligned}
$$




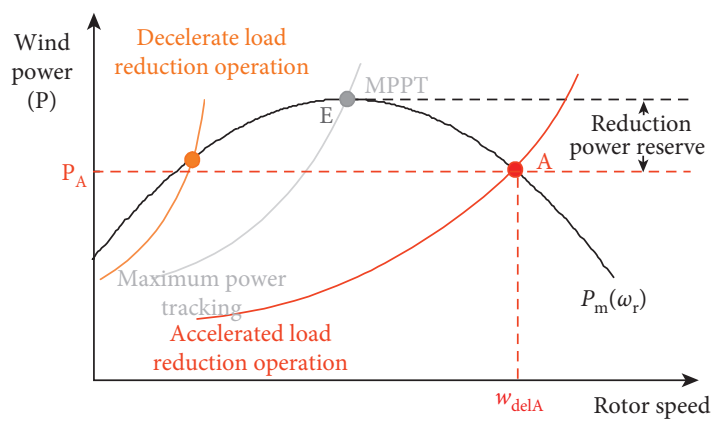

FIgURE 1: Power tracking curve of the fan under load reduction operation.

\section{Wind Turbine Active Participation in Microgrid Frequency Control Strategy Based on Model Predictive Control}

The model predictive control system is introduced in Section 3 . The active control strategy of wind turbines with frequency modulation capacity proposed in this section is based on the model predictive control and frequency response model of the microgrid.

3.1. Frequency Response Model of Microgrid with Wind Turbine Actively Participating in Frequency Control. The traditional active power control strategy of the wind turbine is based on the maximum power tracking curve generation success rate, so the frequency change and active power injection can be decoupled subtly. The objective of the auxiliary frequency controller in the wind turbine is to support the system frequency by associating its active power output with the system frequency. Therefore, the frequency deviation signal is introduced into the auxiliary frequency control strategy based on model predictive control.

The wind turbine has frequency modulation capability under load shedding mode, and its frequency response model structure is shown in Figure 2. The frequency response model (FRM) of wind turbines participating in frequency control is composed of the microgrid frequency control model and wind turbine model under load shedding operation mode. Its structure diagram is shown in Figure 3.

In Figure 2, the output wind power of the wind turbine can be expressed as the sum of control power command output by the model predictive controller and wind power output according to load shedding power tracking curve:

$$
\Delta P_{\text {wind }}=\Delta P_{f}+\Delta P_{\text {del }} \text {, }
$$

where $\Delta P_{f}$ represents the output power command of the model predictive controller, and $\Delta P_{\text {del }}$ represents the wind power output according to the load shedding power tracking curve.

As shown in Figure 2, the input of the wind turbine frequency response model is the system frequency deviation, and the output is the active power injected into the microgrid. The frequency response model consists of an active power response unit and a rotor speed response unit.

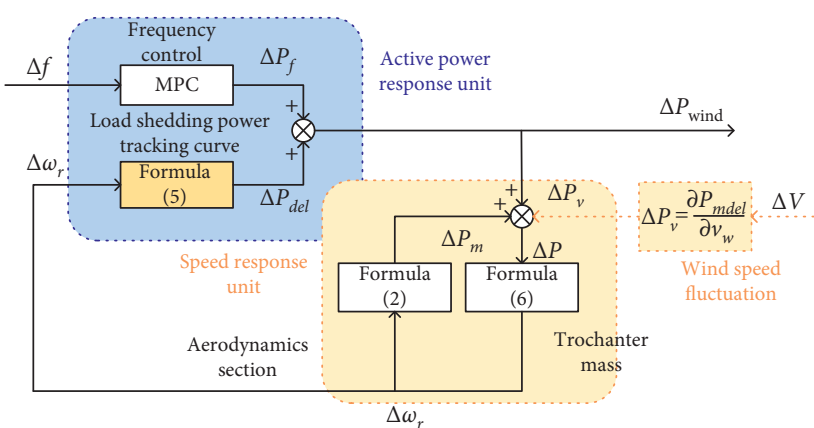

Figure 2: Structural block diagram of the fan frequency response model based on model predictive control.

The active power response unit is composed of two parts: the power response unit of a frequency control strategy based on model predictive control and the rotor speed response unit. Its speed response depends on the rotating dynamics, that is, the balance between the generated electromagnetic power and the mechanical power captured by the fan. The mechanical power captured by the wind turbine is determined according to the fan's mechanical power characteristic curve and rotor speed.

Due to the active participation of wind turbines in frequency control, wind turbines need to operate in load shedding mode to obtain a frequency modulation power margin. In this section, the wind turbine model and the frequency response model of multiarea microgrid under load shedding operation are described and derived. The frequency response model is the basis of the predictive model in the subsequent model predictive control strategy.

According to the mathematical model of wind turbine and microgrid frequency control system under load shedding operation described above, the frequency response model of wind turbine active frequency control is established. The mechanical torque and electromagnetic torque models of the wind turbine are expanded according to Taylor series, and the following mathematical models are obtained:

$$
\left\{\begin{array}{l}
\Delta T_{m}=k_{d e l} k_{w t} \Delta \omega_{t}+k_{d e l} k_{v_{w}} \Delta v, \\
\Delta T_{e}=k_{d e l} k_{w g} \Delta \omega_{t}^{2}, \\
\Delta P_{d e l}=k_{d e l} k_{w g} \Delta \omega_{t}^{3},
\end{array}\right.
$$

where the first-order Taylor series expansion of the linear coefficient around the running point is defined as

$$
k_{w t}=\left.\frac{\partial T_{m}}{\partial \omega_{t}}\right|_{(0)} \quad k_{v w}=\left.\frac{\partial T_{m}}{\partial v_{w}}\right|_{(0)} \quad k_{w g}=\left.\frac{\partial T_{e}}{\partial \omega_{t}}\right|_{(0)} \quad k_{w p}=\left.\frac{\partial P_{e}}{\partial \omega_{t}}\right|_{(0)} .
$$

The mathematical model of the elemental block drive model of the wind turbine under the download operation mode is expressed as an incremental form:

$$
\dot{\omega}_{t}=\frac{k_{d e l} k_{w t}-k_{d e l} k_{w g}-k_{\text {loss }}}{2 H_{w}} \Delta \omega_{t}+\frac{k_{d e l} k_{v w}}{2 H_{w}} \Delta v_{w} .
$$

Taking into account the dynamic relation formula of generator load in the microgrid frequency control model, the 


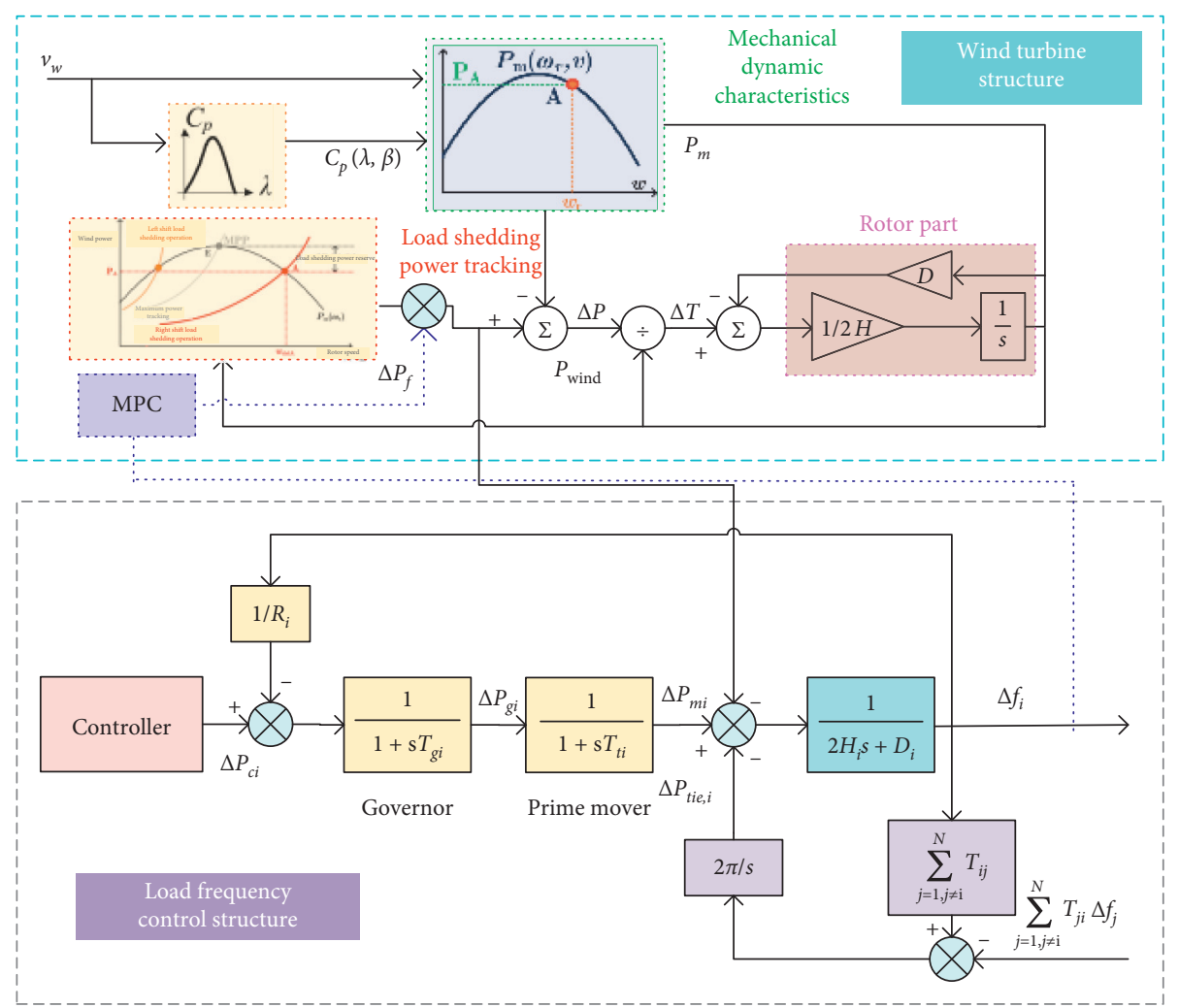

FIGURE 3: Frequency response model block diagram of wind turbine active participation in microgrid frequency control.

following relation between frequency and power can be deduced according to the block diagram of the microgrid frequency response model in which the fan actively participates in the control:

$$
\Delta f_{i}=\left(\Delta P_{\mathrm{mi}}-\Delta P_{L i}-\Delta P_{\text {tiei }}-\Delta P_{\text {wind }}\right) \cdot \frac{1}{2 H_{i} s+D_{i}} .
$$

By combining (7) with (8), and substituting the frequency control model of microgrid described in Section 3 into the above formula, we can obtain

$$
\Delta \dot{f}_{i}=\left(\Delta P_{\mathrm{mi}}-\Delta P_{\mathrm{Li}}-\Delta P_{\mathrm{tiei}}\right) \cdot \frac{1}{2 H_{i}}-k_{d e l} \cdot k_{w p} \cdot \frac{1}{2 H_{i}} \cdot \Delta \omega_{t}-\frac{D_{i}}{2 H_{i}} \cdot \Delta f_{i}-\Delta P_{f} \cdot \frac{1}{2 H_{i}}
$$

By combining formula (11) with the frequency control model of microgrid, the frequency response model of microgrid with wind turbine active participation in frequency control can be uniformly described as the following mathematical model:

$$
\left\{\begin{array}{l}
\dot{x}_{i}(t)=\mathbf{A}_{i} \mathbf{x}_{i}(t)+\mathbf{B}_{i} \mathbf{u}_{i}(t)+\mathbf{F}_{i} \mathbf{w}_{i}(t) \\
\mathbf{y}_{i}(t)=\mathbf{C}_{i} \mathbf{x}_{i}(t)
\end{array}\right.
$$

where $\mathbf{x}_{i}(t)$ is the state variable matrix, $\mathbf{u}_{i}(t)$ is the control variable matrix, $\mathbf{w}_{i}(t)$ is the disturbance variable matrix, $\mathbf{y}_{i}(t)$ is the output vector, and $\mathbf{A}_{i}, \mathbf{B}_{i}, \mathbf{C}_{i}$, and $\mathbf{F}_{i}$ are the coefficient matrices of the state space model.

The regional frequency deviation $\Delta f_{i}$, tie-line power deviation $\Delta P_{t i e, i}$, prime mover output power command $\Delta P_{m i}$, governor output power command $\Delta P_{g i}$, wind turbine rotor speed $\Delta \omega_{t_{i}}$ and synchronous generator power control command $\Delta P_{c i}$ under load shedding operation are selected as state variables. Therefore, the state variable matrix can be expressed as follows:

$$
x_{i}(t)=\left[\begin{array}{llllll}
\Delta f_{i} & \Delta P_{t i e, i} & \Delta P_{m i} & \Delta P_{g i} & \Delta \omega_{t_{i}} & \Delta P_{c i}
\end{array}\right]^{T} .
$$

The output vector $y_{i}(t)$ is the regional control deviation signal $A C E_{i}$. The control variable of the state space model is the output power command $\mathbf{u}_{i}=\Delta P_{f i}$ of the model predictive controller in the wind turbine. In the microgrid controlled by the wind turbine, the power fluctuation and wind speed change of tie-line caused by disturbance variables in other regions are represented as follows:

$$
\mathbf{w}_{i}(t)=\left[\sum_{j=1, j \neq i}^{N} T_{i j} \Delta f_{j} \Delta v_{\omega i}\right]^{T} .
$$

In the frequency response model formula (12), each coefficient matrix is 


$$
\begin{aligned}
& \mathbf{A}_{i}=\left[\begin{array}{cccccc}
-D_{i} /\left(2 H_{i}\right) & -1 /\left(2 H_{i}\right) & 1 /\left(2 H_{i}\right) & 0 & -k_{d e l} K_{w p i} / 2 H_{i} & 0 \\
2 \pi \sum_{j=1, j \neq i}^{N} T_{i j} & 0 & 0 & 0 & 0 & 0 \\
0 & 0 & -1 / T_{t i} & 1 / T_{t i} & 0 & 0 \\
-1 / T_{g i} R_{i} & 0 & 0 & -1 / T_{g i} & 0 & 1 / T_{g i} \\
0 & 0 & 0 & 0 & \kappa / 2 H_{w i} & 0 \\
k_{I} B_{i} & k_{I} & 0 & 0 & 0 & 0
\end{array}\right] \\
& \mathbf{B}_{i}=\left[\begin{array}{c}
1 / 2 H_{i} \\
0 \\
0 \\
0 \\
0 \\
0
\end{array}\right] \quad \mathbf{F}_{i}=\left[\begin{array}{cc}
0 & 0 \\
-2 \pi & 0 \\
0 & 0 \\
0 & 0 \\
0 & -k_{d e l} k_{v m} / 2 H_{w} \\
0 & 0
\end{array}\right], \quad \mathbf{C}_{i}=\left[\begin{array}{c}
\beta_{i} \\
1 \\
0 \\
0 \\
0 \\
0
\end{array}\right],
\end{aligned}
$$

where the parameters $\kappa$ in $\mathbf{A}_{i}$ are

$$
\kappa=k_{d e l} k_{w t}-k_{d e l} k_{w g}-k_{\text {loss }} .
$$

In the frequency response model proposed in formula (12), the dynamic model of wind turbine participating in frequency control under load shedding operation is taken into account. Therefore, the frequency deviation of the system can be obtained by the input of wind speed and various state variables. At the same time, the frequency response model represents the linear relationship between wind speed fluctuation and frequency change, and the system output frequency and other variables are obtained through wind speed data input. Therefore, the frequency response model can be used as a prediction model after transformation, which can provide a reference for the frequency control strategy of wind turbines with frequency regulation capacity.

3.2. Prediction Model of Wind Turbine Active Frequency Control Based on Microgrid System. Firstly, the frequency response model is discretized. The frequency response model of microgrid with wind turbine actively participating in frequency control, namely, the discrete linear state-space model of formula (12), can be expressed as

$$
\left\{\begin{array}{l}
\mathbf{x}_{i}(k+1)=\mathbf{A}_{i, d} \mathbf{x}_{i}(k)+\mathbf{B}_{i, d} \mathbf{u}_{i}(k)+\mathbf{F}_{i, d} \mathbf{w}_{i}(k), \\
\mathbf{y}_{i}(k)=\mathbf{C}_{i, d} \mathbf{x}_{i}(k),
\end{array}\right.
$$

where $\mathbf{A}_{i, d}, \mathbf{B}_{i, d}, \mathbf{C}_{i, d}$, and $\mathbf{F}_{i, d}$ are, respectively, the state coefficient matrix, control coefficient matrix, observation coefficient matrix, and disturbance coefficient matrix after discretization of the continuous state-space model corresponding to formula (12).

According to the principle of the model predictive control algorithm, the system response $\mathbf{y}_{i}\left(k+N_{p} \mid k\right)$ is predicted in the prediction time domain $N_{p}$. Based on the state information $\mathbf{x}_{i}(k+1)$ and the state equation of the current system time (time $k$ ), the system state and output response of the next time $k+1$ are predicted as follows:

$$
\left\{\begin{aligned}
\mathbf{y}_{i}(k+1 \mid k)= & \mathbf{C}_{i, d} \mathbf{A}_{i, d} \mathbf{x}_{i}(k)+\mathbf{C}_{i, d} \mathbf{B}_{i, d} \mathbf{u}_{i}(k \mid k)+\mathbf{C}_{i, d} \mathbf{F}_{i, d} \mathbf{w}_{i}(k \mid k) \\
\mathbf{y}_{i}(k+2 \mid k)= & \mathbf{C}_{i, d} \mathbf{A}_{i, d} \mathbf{x}_{i}(k+1)+\mathbf{C}_{i, d} \mathbf{B}_{i, d} \mathbf{u}_{i}(k+1 \mid k)+\mathbf{C}_{i, d} \mathbf{F}_{i, d} \mathbf{w}_{i}(k+1 \mid k) \\
= & \mathbf{C}_{i, d} \mathbf{A}_{i, d}^{2} \mathbf{x}_{i}(k)+\mathbf{C}_{i, d} \mathbf{B}_{i, d} \mathbf{A}_{i, d} \mathbf{u}_{i}(k \mid k)+\mathbf{C}_{i, d} \mathbf{F}_{i, d} \mathbf{A}_{i, d} \mathbf{w}(k \mid k) \\
& +\mathbf{C}_{i, d} \mathbf{B}_{i, d} \mathbf{u}_{i}(k+1 \mid k)+\mathbf{C}_{i, d} \mathbf{F}_{i, d} \mathbf{w}_{i}(k+1 \mid k), \\
\vdots & \\
\mathbf{y}_{i}\left(k+N_{p} \mid k\right)= & \mathbf{C}_{i, d} \mathbf{A}_{i, d}^{N_{p}} \mathbf{x}_{i}(k)+\mathbf{C}_{i, d} \mathbf{B}_{i, d} \mathbf{A}_{i, d}^{N_{p}-1} \mathbf{u}_{i}(k \mid k)+\cdots \\
& +\mathbf{C}_{i, d} \mathbf{B}_{i, d}\left(\mathbf{A}_{i, d}^{N_{p}-N_{u}}+\cdots+\mathbf{I}\right) \mathbf{u}_{i}\left(k+N_{u}-1 \mid k\right) \\
& +\mathbf{C}_{i, d} \mathbf{F}_{i, d} \mathbf{A}_{i, d}^{N_{p}-1} \mathbf{w}_{i}(k \mid k)+\cdots \\
& +\mathbf{C}_{i, d} \mathbf{F}_{i, d}\left(\mathbf{A}_{i, d}^{N_{p}-N_{u}}+\cdots+\mathbf{I}\right) \mathbf{w}_{i}\left(k+N_{u}-1 \mid k\right),
\end{aligned}\right.
$$


where $N_{u}$ is the control time domain, and the control time domain $N_{u}$ is less than or equal to the prediction time domain $N_{p} ; \mathbf{y}_{i}(k+j \mid k)$ is the output response of the system predicted at time $k$ at time $k+j$, and $j=1,2, \cdots, N_{p} ; \mathbf{u}_{i}(k+$ $j \mid k)$ is the optimal control variable of the system calculated at time $k$ at time $k+j$, and $j=1,2, \cdots, N_{u}-1 ; \boldsymbol{I}$ is the identity matrix; when the time is between the control time domain and the prediction time domain, the optimal control sequence is the control sequence in the last control time domain.

The matrix form of formula (18) can be expressed as

$$
\mathbf{Y}_{i}=\mathbf{G}_{i} \mathbf{x}_{i}(k)+\mathbf{L}_{i} \mathbf{U}_{i}+\mathbf{M}_{i} \mathbf{W}_{i} .
$$

In the formula,

$$
\begin{aligned}
& \mathbf{Y}_{i}=\left[\mathbf{y}_{i}(k+1 \mid k), \mathbf{y}_{i}(k+2 \mid k), \cdots, \mathbf{y}_{i}\left(k+N_{p} \mid k\right)\right]^{T}, \\
& \mathbf{U}_{i}=\left[\mathbf{u}_{i}(k \mid k), \mathbf{u}_{\mathbf{i}}(k+1 \mid k), \cdots, \mathbf{u}_{i}\left(k+N_{u}-1 \mid k\right)\right]^{T}, \\
& \mathbf{G}_{i}=\left[\mathbf{C}_{i, d} \mathbf{A}_{i, d}, \mathbf{C}_{i, d} \mathbf{A}_{i, d}^{2}, \cdots, \mathbf{C}_{i, d} \mathbf{A}_{i, d}^{N_{p}}\right]^{T}, \\
& \mathbf{L}_{i}=\left[\begin{array}{cccc}
\mathbf{C}_{i, d} \mathbf{B}_{i, d} & 0 & \cdots & 0 \\
\mathbf{C}_{i, d} \mathbf{A}_{i, d} \mathbf{B}_{i, d} & \mathbf{C}_{i, d} \mathbf{B}_{i, d} & \cdots & 0 \\
\vdots & \vdots & \cdots & \vdots \\
\mathbf{C}_{i, d} \mathbf{A}_{i, d}^{N_{p}-1} \mathbf{B}_{i, d} & \mathbf{C}_{i, d} \mathbf{A}_{i, d}^{N_{p}-2} \mathbf{B}_{i, d} & \cdots & \xi
\end{array}\right], \\
& \mathbf{M}_{i}=\left[\begin{array}{cccc}
\mathbf{C}_{i, d} \mathbf{F}_{i, d} & 0 & \cdots & 0 \\
\mathbf{C}_{i, d} \mathbf{A}_{i, d} \mathbf{F}_{i, d} & \mathbf{C}_{i, d} \mathbf{F}_{i, d} & \cdots & 0 \\
\vdots & \vdots & \cdots & \vdots \\
\mathbf{C}_{i, d} \mathbf{A}_{i, d}^{N_{p}-1} \mathbf{F}_{i, d} & \mathbf{C}_{i, d} \mathbf{A}_{i, d}^{N_{p}-2} \mathbf{F}_{i, d} & \cdots & \zeta
\end{array}\right] \text {, }
\end{aligned}
$$

where $\xi=\mathbf{C}_{i, d}\left(\mathbf{A}_{i, d}^{N_{p}-N_{u}}+\cdots+\mathbf{I}\right) \mathbf{B}_{i, d}$ in the control coefficient matrix $\mathbf{L}_{i}$, and $\zeta=\mathbf{C}_{i, d}\left(\mathbf{A}_{i, d}^{N_{p}-N_{u}}+\cdots+\mathbf{I}\right) \mathbf{F}_{i, d}$ in the measurement coefficient matrix $\mathbf{M}_{i}$.

Formula (19) is the microgrid frequency control prediction model of the region $I$, and is the prediction unit of model prediction control. Using this model, the frequency and state information of the system can be obtained in advance according to the current output signal, interference signal, and control signal of the system. The forecast information will be used to optimize the wind power output of the wind turbine by using the controller unit of the wind turbine under reduced load operation.

\subsection{Active Frequency Control Strategy of Wind Turbine Based} on Model Predictive Control. The function of active frequency control of wind turbines based on model predictive control is to predict the system output and adjust the power control signal to change the fan output power, so as to obtain the better dynamic frequency response of the system. Therefore, model predictive control needs to determine appropriate control objectives and constraints and also needs to take into account the special dynamic characteristics of wind turbine under load shedding operation.

Figure 4 describes the flow chart structure of the wind turbine model predictive frequency control method. The

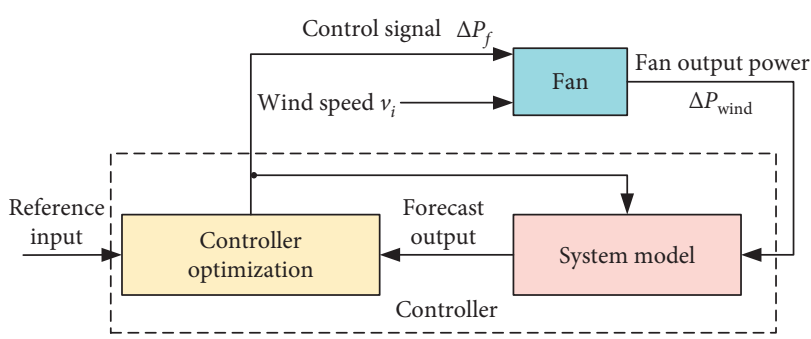

FIGURE 4: Flow chart of the model predictive control method for active frequency control of wind turbine.

model predictive control algorithm uses the predictive model to predict the frequency deviation and state variables of the system and then optimizes the wind turbine power control command sequence in the prediction time domain by optimizing the objective function of the controller. The predictive time domain only selects the control actions in the control time domain of the optimal control vector. In this cycle, the prediction of the previous time is repeated in the next cycle to recalculate the control action sequence.

For microgrid systems with wind turbines actively participating in frequency control under load shedding operation, the goal of model predictive control is to optimize the output power of wind turbines based on the system frequency standard and the actual frequency of the system. Therefore, for each control area containing wind turbines, the control objective function of model predictive control design should not only take into account the dynamic performance of wind turbines, but also consider the minimization of the frequency deviation of the microgrid. By optimizing the target, the proposed strategy can achieve maximum security and economic profits at the same time. The objective function of area $i$ with load shedding wind turbines is expressed as follows:

$$
J_{i}(k)=\sum_{n=1}^{N_{p}}\left[\mathbf{y}_{i}^{T}(k+n \mid k) \mathbf{Q}_{i} \mathbf{y}_{i}(k+n \mid k)+\mathbf{u}_{i}(k+n \mid k)_{i}^{T} \mathbf{R}_{i} \mathbf{u}_{i}(k+n \mid k)\right],
$$

where $\mathbf{Q}_{i}$ and $\mathbf{R}_{i}$ are symmetric weighted positive definite matrices.

The optimal control problem at time $k$ can be expressed as the following optimal control objectives:

$$
\min _{u_{i}(k+n \mid k)} J_{i}(k)
$$

Its constraint condition is the discretization equation of the system frequency response model, namely, the equality constraint:

$$
\left\{\begin{array}{l}
\mathbf{x}_{i}(k+1)=\mathbf{A}_{i, d} \mathbf{x}_{i}(k)+\mathbf{B}_{i, d} \mathbf{u}_{i}(k)+\mathbf{F}_{i, d} \mathbf{w}_{i}(k), \\
\mathbf{y}_{i}(k)=\mathbf{C}_{i, d} \mathbf{x}_{i}(k)
\end{array}\right.
$$

The inequality constraint is the limitation of the frequency modulation capacity margin of the wind generator:

$$
\Delta P_{f} \leq\left(1-k_{d e l}\right) \Delta P_{\text {mmax }} .
$$


Optimization objective formula (22) and constraint condition formula (23) and formula (24) form a programming problem. By using a rolling optimization algorithm, the optimal control action in the control time domain in the control sequence is taken as the actual control action of the system. At the next moment, the system state is remeasured, the optimization process is repeated, and the above steps are repeated. According to the prediction model formula (19), the fan controller in load reduction operation predicts the future state at time $k$ and calculates the optimal control action sequence within the control range. At time $k+1$, the optimization object formula (22) is solved in each controller based on the prediction information. The fan active frequency control strategy for model-based predictive control can be summarized by the following algorithm flow shown in Figure 5.

The first step (initialization): set the initial time $t=k$, the control time domain is $N u$, the prediction time domain is $N_{p}$, the control signal $\boldsymbol{u}_{i}(k)$ at the current time is obtained, and the predicted state variable $\boldsymbol{x}_{i}(k)$ at the previous time is read;

The second step (prediction): the system state $\mathrm{xi}(k+1)$ and output response yi $(k+1)$ of the next time $k+1$ are predicted by using the system control variables and state variables of the current time $k$;

The third step (optimization): by solving the optimization objective formula (22), the control variable $u_{i}(k+1)$ at the next moment of $k+1$ is obtained;

The fourth step (judgment): if the optimal control action of time $k+1$ satisfies the constraint conditions, the controller applies the control action at that time; otherwise, it returns to the second step;

The fifth step (prediction): predict the system state and output response in the next prediction time domain;

The sixth step (implementation): apply the control action $u_{i}(k)$ at time $k+1$, set the time as $k+2$, and go to the second step to execute the predictive control in the next control time domain.

Through the above steps, the control action sequence can be solved by the model predictive controller designed in this section, and then the control signal adjusts the output power of the wind turbine with frequency modulation capacity according to the fluctuation of wind speed, so as to balance the fluctuation of system load and stabilize the system frequency.

\section{The Simulation Analysis}

In order to verify the effectiveness of the proposed model predictive control based wind turbine active participation frequency control strategy in improving the system frequency performance, this section carries out simulation experiments on the four-zone microgrid frequency control model shown in Figure 6. The model considers the frequency state of the synchronous generator and unloaded wind generator in the microgrid to test the proposed fan active frequency control strategy based on model predictive control. The simulation results of wind turbine active participation frequency control strategy based on model predictive control and conventional PID microgrid frequency control strategy proposed in this section are analyzed and compared.

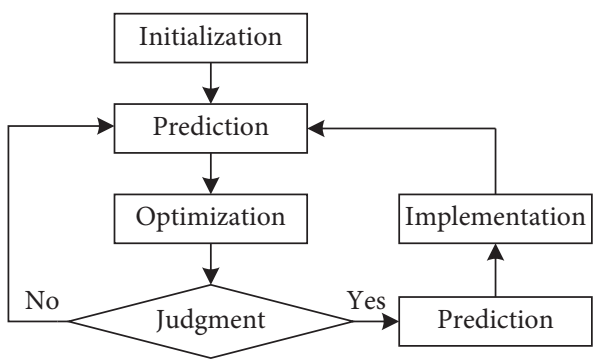

FIGURE 5: Flow chart of the fan active frequency control strategy for MPC.

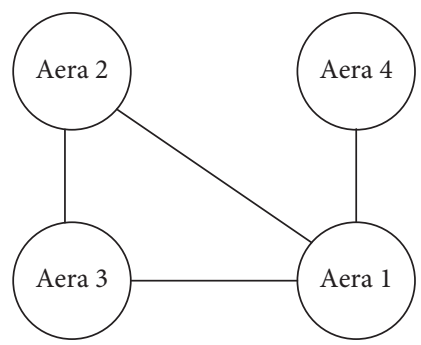

FIGURE 6: Structure of the studied four-zone power system.

In the single-area microgrid system of an airport island in reference [2], the wind turbine is considered as the new energy source, and the proposed active frequency control strategy is used for simulation. The state-space model is shown in the appendix. The parameters of the generator, governor, and non-reheat steam turbine are given in Table 1, which are widely used in references [15-18]. The single-zone system includes the wind turbine model under load shedding operation mode, and its parameters are shown in Table 2. Sampling time $T=0.1 \mathrm{~s}$, prediction time $T_{p}=0.3 \mathrm{~s}$, and control time $T_{c}=0.1 \mathrm{~s}$ were set to simulate the proposed control strategy.

Input the 50-second wind speed sequence as shown in Figure $7(\mathrm{a})$ of standard wind speed data into the wind turbine running in load reduction mode. Figure $7(\mathrm{~b})$ is the wind power output of the wind turbine. Figure 7 (c) and Figure $7(d)$, respectively, show the dynamic response of system frequency deviation and the control variable of wind turbine output power. Table 3 shows the ITAE index values of regional maximum frequency deviation and frequency deviation when using the active participation frequency control strategy and PID frequency control strategy proposed in this paper, respectively. From the comparison of the simulation results in Table 3, the frequency deviation of the active frequency control strategy based on model predictive control is obviously less than that of the PID frequency control strategy.

In Figures 7(b) and 7(c), the red solid line corresponds to the proposed active frequency control strategy based on model predictive control, and the blue dotted line corresponds to the traditional PID control method, and the PID controller parameters are the parameters applied in reference [19]. The blue dotted line shown in Figure 7(b) shows the output wind power of the wind turbine when the 
TABLE 1: Parameters of single-zone microgrid system with non-reheat generators.

\begin{tabular}{lccc}
\hline$T_{t}$ & $T_{g}$ & $H$ & $D$ \\
\hline 0.4 & 0.08 & 0.08335 & 0.015 \\
\hline
\end{tabular}

TABLE 2: Parameters of wind turbine.

\begin{tabular}{lcc}
\hline Fan parameters & Symbol & Value \& units \\
\hline Radius of rotor & $R$ & $38.5 \mathrm{~m}$ \\
Air density & $\mathrm{P}$ & $1.901 \times 10^{-3}(\mathrm{~m} / \mathrm{s})^{-3}$ \\
Number of pole pairs & $P$ & 2 \\
Generator inertia constant & $H_{\text {DFIG }}$ & $5.28 \mathrm{~s}$ \\
Nominal frequency & $f_{\text {nom }}$ & $50 \mathrm{~Hz}$ \\
Based power & $P_{\text {base }}$ & $1.5 \mathrm{MW}$ \\
Load shedding factor & $k_{\text {del }}$ & 0.85 \\
\hline
\end{tabular}

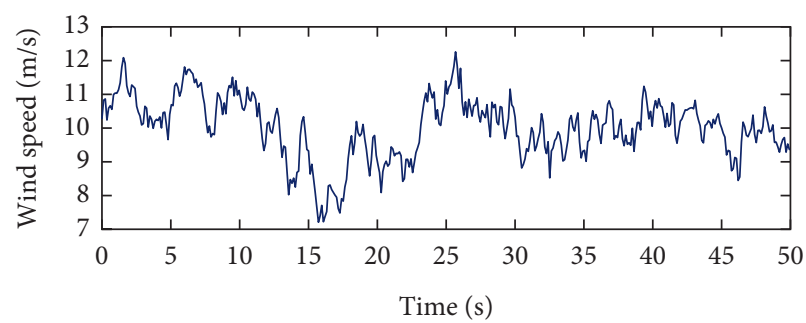

(a)

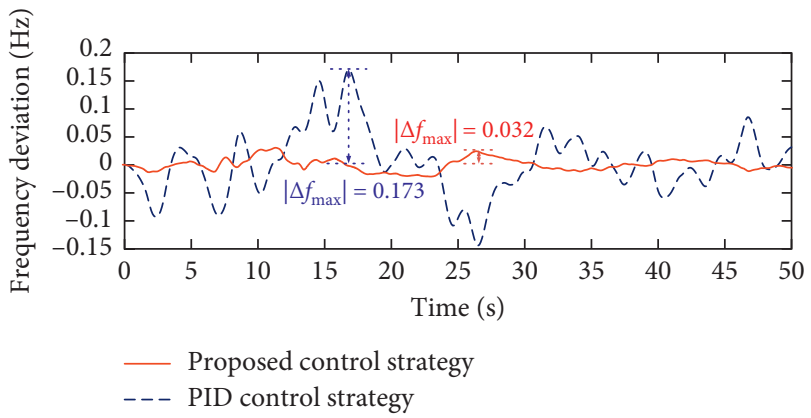

(c)

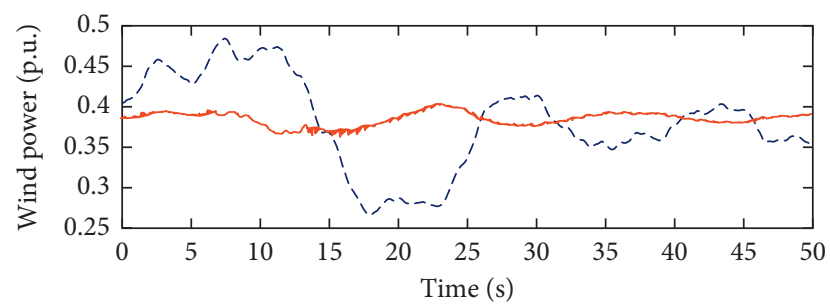

_ Proposed control strategy

-- - PID control strategy

(b)

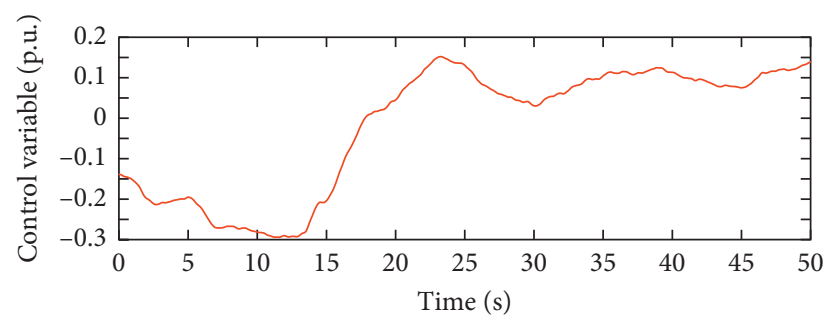

(d)

FiguRE 7: Simulation results of fan active frequency control strategy based on model predictive control. (a) A 50-second wind speed sequence. (b) Wind power output of wind turbine. (c) System frequency deviation. (d) Fan output power command, i.e., control signal.

traditional PID load frequency control method is adopted, and the red solid line is the wind power output of the wind turbine operating in the load shedding mode proposed in this paper. Through comparison, it is easy to know that the wind turbine output power under the load shedding mode proposed in this paper is gentler. As shown in Figure 7(c), the blue dotted line is the frequency fluctuation curve after adopting the PID controller, and the system frequency will be significantly affected by wind power fluctuation. In contrast, the active frequency control strategy based on model predictive control is shown in the red curve in the figure. The frequency dynamic response is better, the system frequency deviation is significantly reduced, and the frequency dynamic response is improved.
TABLE 3: Comparison of single region simulation results.

\begin{tabular}{lcc}
\hline & PID control strategy & Proposed control strategy \\
\hline$\left|\Delta f_{\max }\right|$ & $0.173 \mathrm{~Hz}$ & $0.032 \mathrm{~Hz}$ \\
ITAE & 2.114 & 0.440 \\
\hline
\end{tabular}

In the microgrid system of an airport in reference [2], considering that the four terminals are relatively independent, the wind turbine active participation frequency control strategy proposed in this paper is simulated and tested on the four-area microgrid frequency control model. The system structure is the same as the four-area simulation structure in paper three, and its state-space model is shown in the appendix. The simulation rated parameters of four-zone 
TABLE 4: Simulation rated parameters of four-zone microgrid with non-reheat prime mover.

\begin{tabular}{lcccc}
\hline & $T_{t}$ & $T_{g}$ & $H$ & $D$ \\
\hline Area 1 & 0.4 & 0.08 & 0.08335 & 0.015 \\
Area 2 & 0.33 & 0.072 & 0.111 & 0.04 \\
Area 3 & 0.35 & 0.07 & 0.08 & 0.05 \\
Area 4 & 0.375 & 0.085 & 0.065 & 0.0667 \\
\hline
\end{tabular}

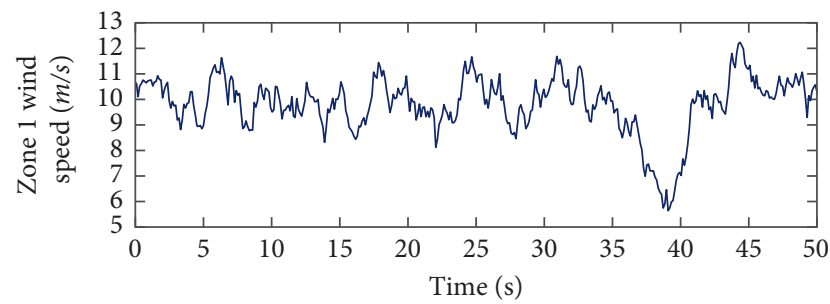

(a)

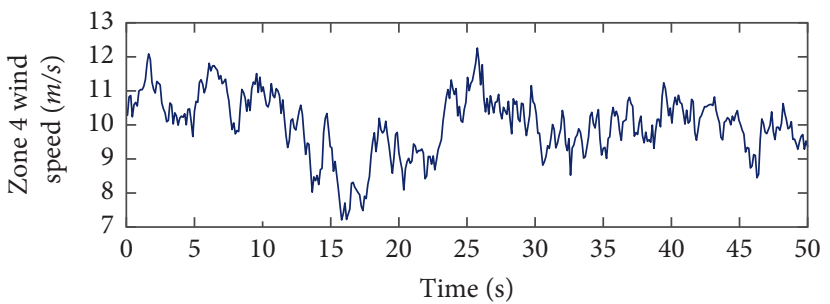

(b)

FIgURE 8: Wind speed series curve. (a) $50 \mathrm{~s}$ wind speed sequence input in zone 1. (b) $50 \mathrm{~s}$ wind speed sequence input in zone 4.

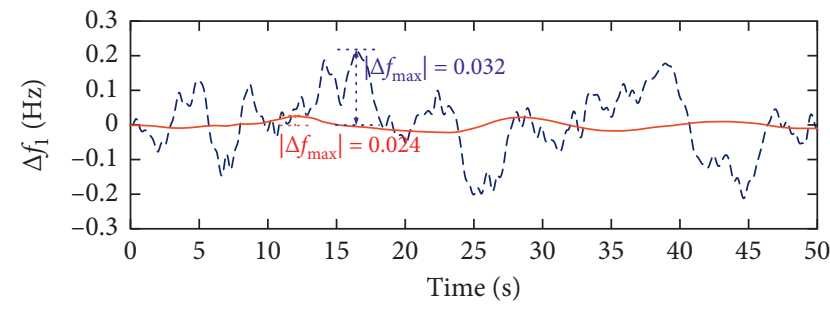

_ Proposed control strategy - - - PID control strategy

(a) 1

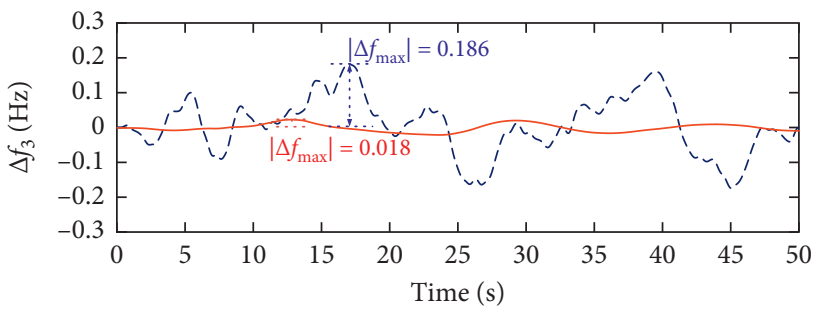

- Proposed control strategy - - - PID control strategy

(c)

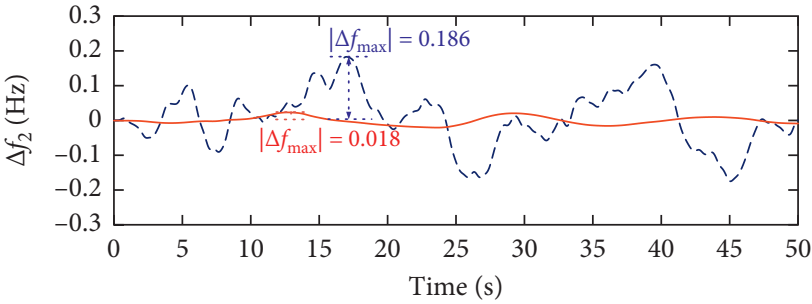

_ Proposed control strategy - - - PID control strategy

(b)

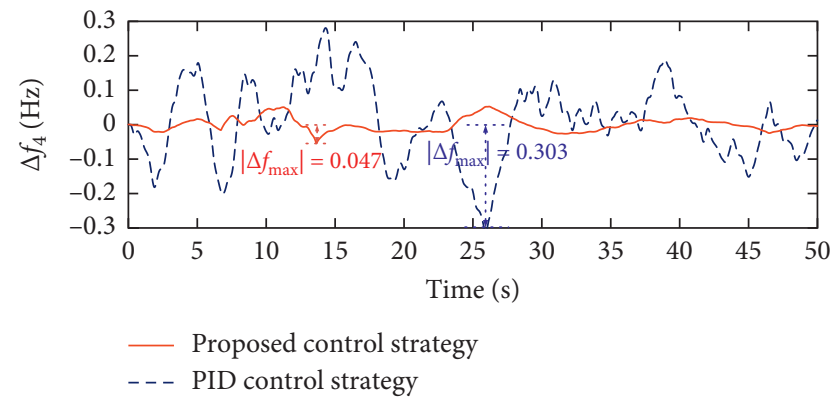

(d)

FIGURE 9: Frequency deviation of four regions.

microgrid with non-reheat prime mover are shown in Table 4 . In order to verify the effectiveness of the proposed strategy in improving the frequency performance of the system, wind turbine models operating in load shedding mode are also added in zone 1 and zone 4 . The parameters of the wind turbine are the same as those used in Section 4.1. The standard wind speed series of 50 seconds is input into the wind turbine of area 1 and area 4 , respectively, as shown in Figures 8(a) and 8(b). At the same time, when the prediction time domain is $T_{p}=0.3 \mathrm{~s}$, and the control time domain is $T_{c}=0.1 \mathrm{~s}$, the proposed scheme is simulated.
Similarly, the proposed wind turbine active participation in the microgrid frequency control strategy is compared with the traditional PID microgrid frequency control strategy in this section. The frequency deviation of zone 1 to zone 4 is shown in Figure 9, and the ITAE index values of frequency deviation and maximum frequency deviation of each region are given in Tables 5 and 6 respectively.

As shown in Figure 9, Tables 5 and 6, the results show that the strategy proposed in this paper can well control the output power of the wind turbine to make it output appropriate power to suppress the frequency fluctuation. 
TABLE 5: The maximum frequency deviation of each region.

\begin{tabular}{lcc}
\hline Section number & PID control strategy & Proposed control strategy \\
\hline Area 1 & $0.213 \mathrm{~Hz}$ & $0.024 \mathrm{~Hz}$ \\
Area 2 & $0.186 \mathrm{~Hz}$ & $0.018 \mathrm{~Hz}$ \\
Area 3 & $0.186 \mathrm{~Hz}$ & $0.018 \mathrm{~Hz}$ \\
Area 4 & $0.303 \mathrm{~Hz}$ & $1.47 \mathrm{~Hz}$ \\
\hline
\end{tabular}

TABLE 6: Frequency deviation ITAE index values in each region.

Section number PID control strategy Proposed control strategy

\begin{tabular}{lll}
\hline Area 1 & 3.7 & 0.5 \\
Area 2 & 3.2 & 0.4 \\
Area 3 & 3.2 & 0.4 \\
Area 4 & 4.5 & 0.8 \\
\hline
\end{tabular}

Therefore, compared with the PID frequency control method, the system frequency deviation of fan active frequency control strategy based on model predictive control is extremely small. At the same time, the frequency response of each area has good dynamic performance no matter there is a wind turbine connected or not. This shows that the proposed fan active participation frequency control strategy of wind turbines based on model predictive control can provide control instructions for wind turbines and help them output appropriate wind power to balance the frequency fluctuation of the system. The local frequency variation of each region converges to zero to ensure that the frequency deviation of each region is kept within the safe working range. Similarly, the model predictive control strategy of the wind turbine needs input wind speed information to predict the state variables and output variables of the system, but in fact, it is difficult to obtain accurate wind speed series by prediction. It is necessary to make use of the inertia part of the transmission system in the fan to cause the time delay of its output wind power relative to the real-time wind speed input. This time delay is used to obtain the output power of the wind turbine in advance, so as to effectively control the output wind power of the wind turbine without wind speed prediction, thus enhancing the ability of the microgrid system to effectively smooth the system frequency fluctuation caused by wind turbine access.

\section{Conclusion}

In this paper, an active frequency control strategy of the wind turbine is proposed based on model predictive control by using the power margin of wind turbines operating in load shedding mode. Specifically, the frequency response model of the microgrid system with the load shedding of the wind turbine is utilized to predict the output power and system frequency deviation of the wind turbine. According to the prediction information, the output power control signal of the model predictive controller in the wind turbine can be optimized. On this basis, an MPC-based active frequency control strategy is designed for the wind turbine by rolling prediction and optimization. The wind turbine power control signal after the strategy is used to adjust the output power of the wind turbine and balance the change of the active power of the system to reduce the frequency deviation. Besides, controlled by the proposed strategy, the frequency regulation capacity of the load shedding fan can be fully utilized when the wind speed changes, the frequency deviation can be controlled within the safe operation range, and the dynamic performance of the system frequency can be adjusted to the expected results.

Moreover, due to the power output delay caused by the inertia link of the wind turbine, the proposed strategy is feasible without wind speed prediction, so the strategy can make full use of the advantages of MPC. In order to verify the effectiveness of the strategy, the traditional PID frequency control strategy and the strategy proposed in this paper are simulated and analyzed in a single-area and fourarea microgrid.

\section{Data Availability}

The data used to support the findings of this study are available from the corresponding author upon request.

\section{Conflicts of Interest}

The authors declare that there are no conflicts of interest regarding the publication of this paper.

\section{Acknowledgments}

This study is financially supported by the National Natural Science Foundation of China (No. 71804177) and the Natural Science Foundation of Hunan Province (No. 2019JJ50455).

\section{References}

[1] C. Wang, Y. Mi, Y. Fu, and P. Wang, "Frequency control of an isolated micro-grid using double sliding mode controllers and disturbance observer," IEEE Transactions on Smart Grid, vol. 9, no. 2, pp. 923-930, 2016.

[2] N. Nguyen and J. Mitra, "An analysis of the effects and dependency of wind power penetration on system frequency regulation," IEEE Transactions on Sustainable Energy, vol. 7, no. 1, pp. 354-363, 2015.

[3] M. F. M. Arani and E. F. El-Saadany, "Implementing virtual inertia in DFIG-based wind power generation," IEEE Transactions on Power Systems, vol. 28, no. 2, pp. 1373-1384, 2012.

[4] I. D. Margaris, S. A. Papathanassiou, N. D. Hatziargyriou, A. D. Hansen, and P. Sorensen, "Frequency control in autonomous power systems with high wind power penetration," IEEE Transactions on Sustainable Energy, vol. 3, no. 2, pp. 189-199, 2012.

[5] Q. Chen, H. Shi, and M. Sun, "Echo state network based backstepping adaptive iterative learning control for strictfeedback systems: an error-tracking approach," IEEE Transactions on Cybernetics, vol. 50, no. 7, pp. 1-14, 2020.

[6] M. F. Arani and Y. A.-R. I. Mohamed, "Analysis and impacts of implementing droop control in DFIG-based wind turbines on microgrid/weak-grid stability," IEEE Transactions on Power Systems, vol. 30, no. 1, pp. 385-396, 2015.

[7] Y. Wang, J. Meng, X. Zhang, and L. Xu, "Control of PMSGbased wind turbines for system inertial response and power 
oscillation damping," IEEE Transactions on Sustainable Energy, vol. 6, no. 2, pp. 565-574, 2015.

[8] C. Luo and B.-T. Ooi, "Frequency deviation of thermal power plants due to wind farms," IEEE Transactions on Energy Conversion, vol. 21, no. 3, pp. 708-716, 2006.

[9] M. F. M. Arani and Y. A.-R. I. Mohamed, "Cooperative control of wind power generator and electric vehicles for microgrid primary frequency regulation," IEEE Transactions on Smart Grid, vol. 9, no. 6, pp. 5677-5686, 2017.

[10] Y. Tan, L. Meegahapola, and K. M. Muttaqi, “A suboptimal power-point-tracking-based primary frequency response strategy for DFIGs in hybrid remote area power supply systems," IEEE Transactions on Energy Conversion, vol. 31, no. 1, pp. 93-105, 2015.

[11] L. Holdsworth, J. B. Ekanayake, and N. Jenkins, "Power system frequency response from fixed speed and doubly fed induction generator-based wind turbines," Wind Energy, vol. 7, no. 1, pp. 21-35, 2004.

[12] L.-R. Chang-Chien, W.-T. Lin, and Y.-C. Yin, "Enhancing frequency response control by DFIGs in the high wind penetrated power systems," IEEE Transactions on Power Systems, vol. 26, no. 2, pp. 710-718, 2010.

[13] Y. Liu, L. Jiang, Q. Wu, and X. Zhou, "Frequency control of DFIG-based wind power penetrated power systems using switching angle controller and AGC," IEEE Transactions on Power Systems, vol. 32, no. 2, pp. 1553-1567, 2016.

[14] Q. Chen, S. Xie, M. Sun, and X. He, "Adaptive nonsingular fixed-time attitude stabilization of uncertain spacecraft," IEEE Transactions on Aerospace and Electronic Systems, vol. 54, no. 6, pp. 2937-2950, 2018.

[15] C. Wei, M. Benosman, and T. Kim, "Online parameter identification for state of power prediction of lithium-ion batteries in electric vehicles using extremum seeking," International Journal of Control, Automation and Systems, vol. 17, no. 11, pp. 2906-2916, 2019.

[16] M. Kang, E. Muljadi, K. Hur, and Y. C. Kang, "Stable adaptive inertial control of a doubly-fed induction generator," IEEE Transactions on Smart Grid, vol. 7, no. 6, pp. 2971-2979, 2016.

[17] L. Wu and D. G. Infield, "Towards an assessment of power system frequency support from wind plant-modeling aggregate inertial response," IEEE Transactions on Power Systems, vol. 28, no. 3, pp. 2283-2291, 2013.

[18] F. Cheng, L. Qu, W. Qiao, C. Wei, and L. Hao, "Fault diagnosis of wind turbine gearboxes based on DFIG stator current envelope analysis," IEEE Transactions on Sustainable Energy, vol. 10, no. 3, pp. 1044-1053, 2019.

[19] M. F. M. Arani and Y. A.-R. I. Mohamed, "Dynamic droop control for wind turbines participating in primary frequency regulation in microgrids," IEEETransactions on Smart Grid, vol. 9, no. 6, pp. 5742-5751, 2017. 